\title{
CONSTITUTIVELY OVEREXPRESSED TYPE I INTERFERON TRANSGENIC MEDAKA FISH DOWN-REGULATE THE ANTIVIRAL RESPONSE
}

\author{
Shun Maekawa ${ }^{1}$, Chu-Fang Lo ${ }^{2}$, Han-Ching Wang ${ }^{1}$, Takashi Aoki ${ }^{1,}$ \\ ${ }^{1}$ Institute of Biotechnology, College of Bioscience and Biotechnology, National Cheng Kung \\ University, Tainan, Taiwan \\ ${ }^{2}$ Institute of Bioinformatics and Biosignal Transduction, College of Bioscience and \\ Biotechnology, National Cheng Kung University, Tainan, Taiwan
}

\begin{abstract}
Type I interferon (IFN) is one of most important cytokines for antiviral responses in fish innate immunity. Expression of IFN is induced following pattern recognition of virus and activates transcription of various IFN-stimulated genes (ISGs) to promote antiviral activity. However, level of expression of IFN is strictly regulated. In a normal state, low amounts of IFN are constitutively secreted, which are important for maintenance of appropriate antiviral responses. However, longterm and excessive IFN-stimulation resulted in desensitization of IFN (to prevent inappropriate responses and autoimmunity). Desensitization of IFN and its physiologic role for constitutive IFN in normal state are not well characterized in fish. We previously identified two types of type I IFN from medaka fish (Oryzias latipes), designated olIFNa and olIFNd (Maekawa et al., Fish Shellfish Immunol, 2015). To investigate effects of excessive type I IFN stimulation in vivo, we generated and characterized transgenic medaka fish that constitutively expressed the ollFNd gene (ollFNd$\mathrm{Tg}$ ). We constructed a dual promoter expression vector for overexpression of ollFNd under an EF $1 \alpha$ promoter and a DsRed reporter gene under control of $\gamma \mathrm{F}$-crystaline promoter. Embryos injected with vector were raised to sexual maturity and F1 embryos identified by fluorescent microscopy (seeing DsRed expression in lens of eye). In olIFNd-Tg larval fish, expression of interferon-stimulated genes (Mx, GIG1, ISG15 and ISG56) ISGs were downregulated compared to wild-type fish (WT), despite IFNd-overexpression. The IFN signal transduces STAT1b, STAT2 and IRF9 is also down-regulated in olIFNd-Tg. These results indicated desensitization of IFNstimulation. Expression of RIG-I-like receptors (RLR) signal molecules that recognize the virus and induce type I IFN expression were assessed. In olIFNd-Tg, expression levels of MDA5, MITA and IRF3 were lower than WT. Moreover, IFNa (endogenous) expression was also reduced in olIFNd-Tg. Finally, we demonstrated that olIFNd-Tg larval fish had increased viral titers following infection with nervous necrosis virus (NNV) compared to WT. These data indicated that olIFNd-Tg was susceptible for NNV caused by downregulation of RLR signals. In conclusion, constitutive and excessive olIFNd resulted in desensitization of IFN-stimulation which would have be caused by downregulatioon of IFN signal transduces. The phenotype of olIFNd-Tg is susceptible for NNV caused by down-regulation of RLR-IFN signaling. These results suggesting that adequate type I IFN expression in normal state is important to maintain innate immune responses.
\end{abstract}

\section{KEYWORDS}

Type I interferon, Antiviral response, Medaka fish, Transgene, IFN-stimulate genes

§Corresponding author. TEL: +886-6-2757575 ext 65603-810 FAX: +886-6-276-6505

E-mail: takashi.aoki65@gmail.com 